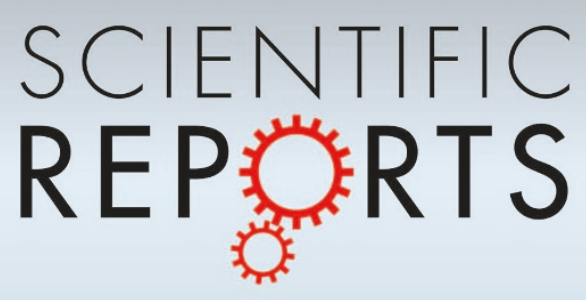

\title{
OPEN An engineered genetic selection for \\ NETWORKS ternary protein complexes inspired by a natural three-component hitchhiker mechanism
}

SUBJECT AREAS:

APPLIED MICROBIOLOGY

SYNTHETIC BIOLOGY

PROTEIN-PROTEIN INTERACTION

Received

22 September 2014

Accepted

2 December 2014

Published

22 December 2014

Correspondence and requests for materials should be addressed to

M.P.D. (md255@ cornell.edu)
Hyeon-Cheol Lee', Alyse D. Portnoff', Mark A. Rocco ${ }^{2}$ \& Matthew P. DeLisa ${ }^{1,2}$

'School of Chemical and Biomolecular Engineering, Cornell University, Ithaca, NY 14853 USA, ${ }^{2}$ Department of Biomedical Engineering, Cornell University, Ithaca, NY 14853 USA.

The bacterial twin-arginine translocation (Tat) pathway is well known to translocate correctly folded monomeric and dimeric proteins across the tightly sealed cytoplasmic membrane. We identified a naturally occurring heterotrimer, the Escherichia coli aldehyde oxidoreductase PaoABC, that is co-translocated by the Tat translocase according to a ternary "hitchhiker" mechanism. Specifically, the PaoB and PaoC subunits, each devoid of export signals, are escorted to the periplasm in a piggyback fashion by the Tat signal peptide-containing subunit PaoA. Moreover, export of PaoA was blocked when either PaoB or PaoC was absent, revealing a surprising interdependence for export that is not seen for classical secretory proteins. Inspired by this observation, we created a bacterial three-hybrid selection system that links the formation of ternary protein complexes with antibiotic resistance. As proof-of-concept, a bispecific antibody was employed as an adaptor that physically crosslinked one antigen fused to a Tat export signal with a second antigen fused to TEM-1 $\beta$-lactamase (Bla). The resulting non-covalent heterotrimer was exported in a Tat-dependent manner, delivering Bla to the periplasm where it hydrolyzed $\beta$-lactam antibiotics. Collectively, these results highlight the remarkable flexibility of the Tat system and its potential for studying and engineering ternary protein interactions in living bacteria.

$\mathrm{T}$ he hallmark of the twin-arginine translocation (Tat) pathway is its unique ability to transport folded proteins across the tightly sealed cytoplasmic membrane (reviewed in ref. 1 and elsewhere). At present, the exact mechanistic details of this process remain poorly understood; however, the versatility of the Tat system is firmly established on the basis of the structural and functional diversity of proteins that transit this pathway. Indeed, Tat substrates range in size between 20 and $70 \AA$ in diameter, but also much smaller in the case of some engineered substrates ${ }^{2}$, and include soluble periplasmic enzymes ${ }^{3-5}$, lipoproteins ${ }^{6}$, and inner and outer membrane proteins $s^{7-9}$.

While the bulk of Tat substrates studied so far are monomeric proteins (e.g., Escherichia coli molybdoenzyme TorA), heterodimeric proteins can also transit this pathway. One notable example is the nickel-iron [NiFe] hydrogenase 2 (HYD2) system of $E$. coli that catalyzes the reversible oxidation of hydrogen and allows bacteria to use hydrogen as an energy source for growth. HYD2 is a heterodimer comprised of a large subunit (HybC), containing the $[\mathrm{NiFe}]$ active site but lacking any discernible export signal, and a small subunit (HybO), bearing iron-sulfur [Fe-S] clusters and also an N-terminal Tat signal peptide. Besides assembling [Fe-S] clusters, the HybO subunit also assembles with the large HybC subunit in the cytoplasm prior to export. Following assembly, the HybOC heterodimer is exported to the periplasm by virtue of the Tat signal peptide on the HybO subunit ${ }^{9}$. This mode of export, whereby one substrate protein devoid of any known export signal is co-translocated in a complex with its signal peptide-bearing partner, is referred to as "hitchhiker" co-translocation".

A handful of additional substrates are predicted to follow the hitchhiker mechanism ${ }^{10-12}$, which has recently inspired new methods for expressing and engineering heterologous proteins. For example, it has been shown that preassembled dimeric proteins, including the covalently linked heavy and light chains of a $\mathrm{F}_{\mathrm{AB}}$ antibody, can be targeted to the periplasm via the hitchhiker route ${ }^{13}$. More recently, hitchhiker-mimetic genetic assays for monitoring and engineering pairwise protein interactions have been reported ${ }^{14,15}$. In these assays, the test protein (i.e., bait or receptor) to be screened is engineered with an $\mathrm{N}$-terminal Tat signal peptide, whereas the known or 
putative partner protein (e.g., prey or ligand) is fused to a reporter enzyme whose co-translocation to the periplasm gives rise to a distinct and quantifiable phenotype. For example, by using mature TEM1 $\beta$-lactamase (Bla) as the reporter enzyme, the binding between a receptor and its ligand can be conveniently linked to antibiotic resistance ${ }^{15,16}$.

To find additional examples of hitchhiker substrates that might spawn similar technology development, we turned our attention to the molybdenum-containing iron-sulfur flavoprotein PaoABC (formerly YagTSR) from E. coli. PaoABC is an aldehyde oxidoreductase that oxidizes a broad spectrum of aldehydes to their respective acids $^{17}$. The $135-\mathrm{kDa}$ enzyme comprises a non-covalent $(\alpha \beta \gamma)$ heterotrimer with a large $(78.1 \mathrm{kDa})$ molybdenum cofactor (Moco)containing PaoC subunit, a medium $(33.9 \mathrm{kDa})$ flavin adenine dinucleotide (FAD)-containing PaoB subunit, and a small $(21.0 \mathrm{kDa})$ $2 \times[2 \mathrm{Fe} 2 \mathrm{~S}]$-containing PaoA subunit ${ }^{18}$. Only the PaoA protein contains an N-terminal signal peptide, which can deliver heterologous proteins to the periplasm via the Tat pathway ${ }^{19}$. The fact that only PaoA carries a Tat signal peptide led to an early hypothesis that the heterotrimeric PaoABC complex may be co-translocated ${ }^{20}$; however, experimental evidence in support of this hypothesis has been lacking. Here, we show that PaoABC is a bona fide Tat substrate. Moreover, the $\mathrm{PaoB}$ and PaoC subunits, which are each devoid of any known export signals, are escorted to the periplasm by PaoA in a piggyback fashion. Akin to HybOC, there is an interdependence between the small, signalpeptide bearing PaoA subunit and the larger PaoB and PaoC subunits for productive membrane translocation of PaoABC.

Drawing inspiration from this three-component hitchhiker mechanism, we developed a genetic selection for studying and engineering ternary protein complexes. Our hypothesis was that a bispecific affinity protein could be used as an adaptor to co-recruit one ligand fused to a Tat export signal and a second ligand fused to a periplasmic reporter protein. To test this notion, a dual antigen-binding protein was created by recombinant fusion of two different single-chain Fv $(\mathrm{scFv})$ antibodies joined by a flexible Gly-Ser linker, thereby yielding a tandem scFv construct. Two additional chimeras were created by fusing the cognate ligand of each $\mathrm{scFv}$ to the Tat-dependent signal peptide of E. coli TorA and mature Bla, respectively. Co-expression of the three chimeras in $E$. coli resulted in the formation of a noncovalent heterotrimer that was exported in a Tat-dependent manner, thereby delivering Bla to the periplasm where it hydrolyzed $\beta$-lactam antibiotics. This new bacterial three-hybrid $(\mathrm{B} 3 \mathrm{H})$ system opens the door to routine selection of ternary protein complexes, especially those involving bispecific affinity proteins, all without the need for purification or immobilization of either binding target.

\section{Results}

Export of PaoABC is Tat dependent and involves a hitchhiker mechanism. E. coli PaoA carries a canonical Tat signal peptide $\mathrm{e}^{10,19}$ and is known to form a heterotrimeric complex with $\mathrm{PaoB}$ and $\mathrm{PaoC}^{17}$. Hence, we hypothesized that PaoABC might be exported out of the cytoplasm by the Tat translocase according to a hitchhiker mechanism whereby $\mathrm{PaoB}$ and PaoC are ferried to the periplasm by the signal peptide-bearing PaoA subunit. To test this notion, the paoABC genes were assembled into a synthetic operon under the control of a single Trc promoter in plasmid pTrc99Y using yeast-based recombineering $^{21}$ (Supplementary Fig. S1). The resulting plasmid, pPaoABC, permits co-expression of the PaoABC subunits each appended with a unique C-terminal tag for specific immunodetection. Following expression of the synthetic paoABC operon in wild-type (wt) MC4100 cells, all three PaoABC subunits were detected in the periplasm, with a comparable amount of each subunit also detected in the cytoplasm (Fig. 1). In B1LK0 cells, which lack the essential TatC component of the translocase ${ }^{22}$, each PaoABC subunit accumulated only in the cytoplasm (Fig. 1), indicating that

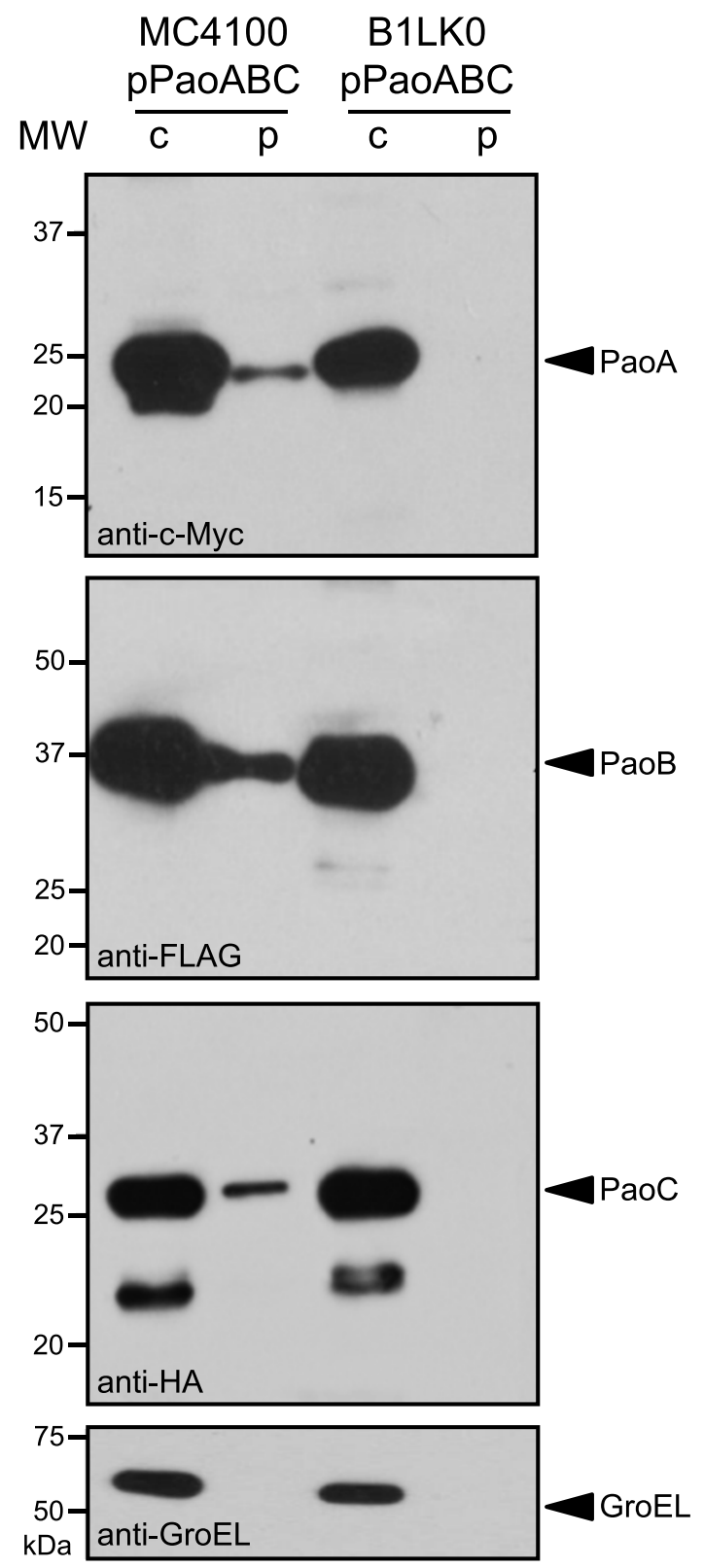

Figure 1 Tat-mediated hitchhiker export of PaoABC heterotrimer. Western blot analysis of cytoplasmic (c) and periplasmic (p) fractions prepared from E. coli MC4100 or B1LK0 cells carrying plasmid pPaoABC, which encodes the synthetic paoABC operon. Fractions were normalized to an equivalent number of cells prior to loading. Co-expression of epitopetagged PaoA-c-Myc, PaoB-FLAG and PaoC-HA were detected using antic-Myc, anti-FLAG, and anti-HA antibodies, respectively. The cytoplasmic chaperone GroEL was detected using anti-GroEL antibody and served as a fractionation marker. Molecular weight (MW) markers are indicated on the left. See Supplementary Fig. S7 for uncropped versions of the images.

transport of PaoABC to the periplasm is dependent on the Tat pathway.

If PaoABC export involves a hitchhiker mechanism, then the absence of PaoA should result in cytoplasmic accumulation of the $\mathrm{PaoB}$ and PaoC subunits even in the presence of a functional Tat translocase. This idea was tested by constructing plasmid $\mathrm{pPao}(\Delta \mathrm{A}) \mathrm{BC}$, which encoded the pao operon but without the gene encoding PaoA. This plasmid was used to transform $\triangle p a o A$ cells or alternatively HL0281 cells (BW25113 $\Delta p a o A B-p a o C::$ Kan) to eliminate possible interference from endogenous Pao proteins. In support 
of our hitchhiker hypothesis, when signal-peptide bearing PaoA was absent, targeting of $\mathrm{PaoB}$ and $\mathrm{PaoC}$ to the periplasm was abolished and both proteins accumulated exclusively in the cytoplasm (Fig. 2A and B).

PaoB and PaoC subunits influence targeting of PaoA. In the case of HybOC export, both HybC and HybO accumulated in the cytoplasm as a precursor when either was expressed in the absence of the other?. This interdependence between the small and large subunits for export signified a unique quality control (QC) mechanism not seen for classical secretory proteins, namely that the folding and proper assembly of the subunits is necessary for translocation to the periplasm. To determine if export of PaoABC was governed by a similar $\mathrm{QC}$ mechanism, we created plasmids $\mathrm{pPaoA}(\Delta \mathrm{B}) \mathrm{C}$ and $\mathrm{pPaoAB}(\Delta \mathrm{C})$, which lacked the gene encoding the $\mathrm{PaoB}$ or $\mathrm{PaoC}$ subunit, respectively, and used these to transform the respective $\triangle p a o B, \Delta p a o C$, or $\triangle p a o A B-p a o C::$ Kan strains. When either the $\mathrm{PaoB}$ or PaoC subunit was absent, the other two subunits including the signal peptide-bearing PaoA protein accumulated in the cytoplasm (Fig. 2A and B). Therefore, while PaoA is required for membrane targeting of $\mathrm{PaoB}$ and $\mathrm{PaoC}$, the presence of the PaoA signal peptide itself is not sufficient for translocation if either of the other subunits is missing, reminiscent of the situation observed for HybOC 9 .

The interdependence among the three subunits highlights the importance of PaoABC heteroassembly in the export process. To confirm complex formation in vivo under the conditions used here, we co-produced $\mathrm{PaoB}$ and $\mathrm{PaoC}$ along with a hexahistidine-tagged version of PaoA in $\triangle p a o A B$-paoC::Kan cells and subjected soluble lysates derived from these cells to $\mathrm{Ni}^{2+}$-affinity chromatography. Immunoblotting revealed that $\mathrm{PaoB}$ and $\mathrm{PaoC}$ co-eluted with the affinity-purified PaoA (Fig. S2A). In contrast, no Pao proteins were detected in elution fractions prepared from cells co-producing PaoB and PaoC in the absence of PaoA (Fig. S2A). We next subjected the same eluted fractions containing the co-purified proteins to blue native polyacrylamide gel electrophoresis (BN-PAGE), a special case of native electrophoresis for high-resolution separation and molecular mass estimation of native proteins and protein complexes ${ }^{23}$. Two distinct bands were observed: a faint band of $\sim 135 \mathrm{kDa}$ corresponding to the expected PaoABC trimer (Fig. S2B). A second more prominent band of $\sim 270 \mathrm{kDa}$ was also observed (Fig. S2B). While the identity of this larger complex was not confirmed, it may correspond to a possible dimer of PaoABC trimers. In further support of complex formation in vivo, gel filtration analysis of the periplasmic fraction derived from $\triangle p a o A B-p a o C::$ Kan cells expressing PaoABC revealed a prominent $135-\mathrm{kDa}$ peak corresponding to the PaoABC trimer (Fig. S2C), indicating that complex formation was not an artifact of the $\mathrm{Ni}^{2+}$-affinity purification process. When the same periplasmic fraction was subjected to BN-PAGE analysis, we again observed bands corresponding to PaoABC $(\sim 135 \mathrm{kDa})$ and also the unidentified $\sim 270-\mathrm{kDa}$ complex (Fig. S2D). Collectively, these data confirm formation of a stable $\alpha \beta \gamma$ heterotrimer in vivo that we suspect may further assemble into a $(\alpha \beta \gamma)_{2}$ complex.

A three-hybrid genetic selection inspired by PaoABC cotranslocation. We previously demonstrated that the two-component hitchhiker mechanism governing HybOC export ${ }^{9}$ could be exploited in a general way for monitoring binary protein interactions in . coli $^{15,16,24}$. Here, we speculated that three-component hitchhiker export by the Tat pathway could be leveraged to create a genetic selection for studying and engineering ternary protein interactions. As proof-of-concept, a bispecific antibody (BsAb) was created by recombinant fusion of two

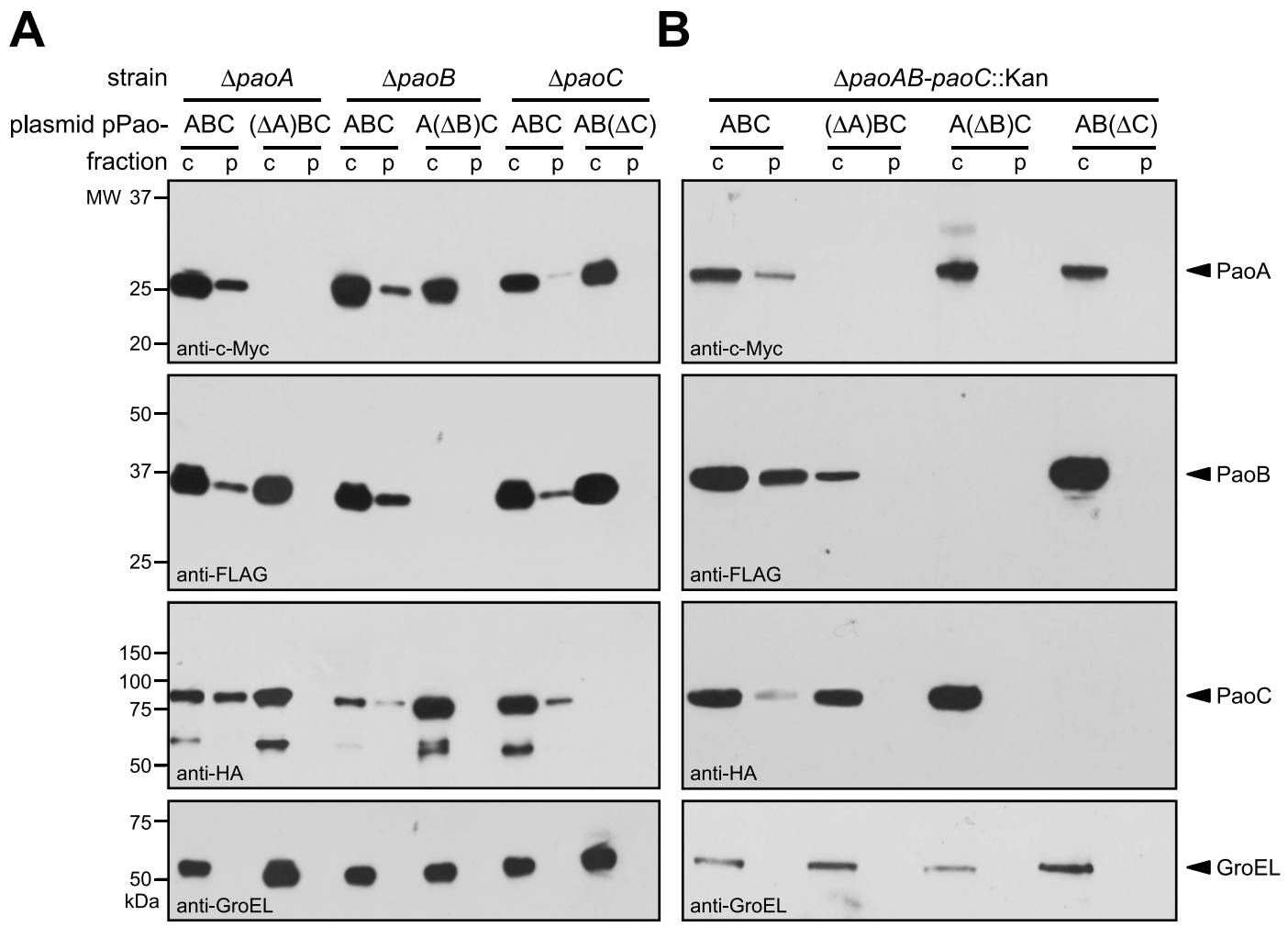

Figure $2 \mid$ Interdependence of three PaoABC subunits for export. Western blot analysis of cytoplasmic (c) and periplasmic (p) fractions prepared from E. coli strain BW25113 and isogenic derivatives lacking individual pao genes (A) or the complete paoABC operon (B). Strains were complimented with plasmid pPaoABC or derivatives of pPaoABC lacking individual subunits as indicated. Fractions were normalized to an equivalent number of cells prior to loading. Co-expression of epitope-tagged PaoA-c-Myc, PaoB-FLAG and PaoC-HA were detected using anti-c-Myc, anti-FLAG, and anti-HA antibodies, respectively. The cytoplasmic chaperone GroEL was detected using anti-GroEL antibody and served as a fractionation marker. Molecular weight (MW) markers are indicated on the left. See Supplementary Fig. S8 for uncropped versions of the images. 
different scFv antibodies joined through a Gly-Ser linker in plasmid pTG-Fv ${ }_{2}-\mathrm{gB}$ (Fig. S3). This tandem $\mathrm{scFv}\left(\mathrm{Fv}_{2}\right)$ design forms the basis of bispecific $T$-cell engager molecules (BiTEs) developed for cancer immunotherapy ${ }^{25}$. We chose two $\mathrm{scFv}$ molecules that were previously optimized for intracellular expression because in our system scFv folding and antigen binding must occur cytoplasmically. Specifically, one of the two Fv fragments was directed against the 47residue basic region-leucine zipper domain of the yeast transcription factor Gcn4 (scFv-GCN4) $)^{15,26}$ and the other was directed against the capsid protein D $(\mathrm{gpD})$ of bacteriophage lambda (scFv-D10) ${ }^{27}$. The cognate antigens for the engineered $\mathrm{Fv}_{2}$ were cloned in the same plasmid (Fig. S3), where each was modified as follows: the Gcn4 leucine zipper domain was modified with an $\mathrm{N}$-terminal Tat signal peptide derived from E. coli TorA (TG) ${ }^{15}$ and gpD was fused to the $\mathrm{N}$-terminus of mature Bla lacking its native export signal (gB). Together, these engineered chimeras form the basis of a $\mathrm{B} 3 \mathrm{H}$ assay, whereby the BsAb creates a physical crosslink between the Tat signal peptide and the Bla reporter enzyme and the subsequent export of this non-covalent heterotrimer confers resistance to $\beta$-lactam antibiotics due to the presence of Bla in the periplasm (Fig. 3A).

Dual antigen-binding activity is faithfully reported by $\mathrm{B} 3 \mathrm{H}$ assay. To evaluate our engineered $\mathrm{B} 3 \mathrm{H}$ assay, E. coli strain MC4100A was transformed with plasmid pTG- $\mathrm{Fv}_{2}-\mathrm{gB}$ and the resulting cells were challenged with varying concentrations of carbenicillin (Carb). Cells co-producing the $\mathrm{TG}, \mathrm{Fv}_{2}$, and $\mathrm{gB}$ fusions from $\mathrm{pTG}-\mathrm{Fv}_{2}-\mathrm{gB}$ showed a much greater resistance to Carb than control cells carrying either an empty plasmid or plasmid pTG-gB, which lacked the $\mathrm{Fv}_{2}$ adaptor (Fig. 3B). To confirm whether the resistant phenotype of MC4100A pTG- $\mathrm{Fv}_{2}$-gB cells depended on the Tat pathway, we mutated the RR motif in the TorA signal peptide to KK, a substitution known to completely block export ${ }^{13}$. Indeed, cells co-producing $\mathrm{T}(\mathrm{KK}) \mathrm{G}$, $\mathrm{Fv}_{2}$, and $\mathrm{gB}$ from $\mathrm{pT}(\mathrm{KK}) \mathrm{G}-\mathrm{Fv}_{2}-\mathrm{gB}$ were sensitive to Carb (Fig. 3B). Likewise, when the original $\mathrm{pTG}-\mathrm{Fv}_{2}-\mathrm{gB}$ was used to transform $\triangle t a t C$ mutant cells, the Carb-resistant phenotype was abolished (Fig. 3B). In all cases involving MC4100A cells, plating on non-selective medium resulted in comparable robust growth indicating that the drug sensitivity observed for control cells was not due to inherent growth defects (Fig. 3B). In the case of B1LK0A cells, there was a moderate growth defect observed in the absence of Carb; however, this decreased resistance was not sufficient to account for the near complete inhibition in the presence of 20 and $50 \mu \mathrm{g} / \mathrm{ml}$ Carb. This, in addition to the results with pT(KK) G- $\mathrm{Fv}_{2}-\mathrm{gB}$ in MC4100A cells, suggested that the strong Carb resistance observed for MC4100A pTG- $\mathrm{Fv}_{2}$-gB cells depended on the Tat pathway.

Immunoblotting of subcellular fractions prepared from MC4100A pTG- $\mathrm{Fv}_{2}-\mathrm{gB}$ cells confirmed that all three chimeras accumulated in the cytoplasm as intact fusion proteins, albeit with some evidence for degradation (Fig. 3C). Consistent with the resistant phenotype observed above, all three intact fusions were detected in the periplasm of these cells. Since TG was the only fusion carrying a signal peptide, we conclude that export of $\mathrm{Fv}_{2}$ and gB must occur by a similar three-component hitchhiker mechanism used by PaoABC. In the absence of the $\mathrm{Fv}_{2}$ adaptor, $\mathrm{gB}$ was no longer detected in the periplasm (Fig. 3C) confirming that export of the Bla-containing chimera depended on heteroassembly. Not surprisingly, TG still localized in the periplasm even when the adaptor was absent (Fig. 3C), indicating that the subunit interdependence seen above for PaoABC export was not an inbuilt feature of this engineered hitchhiker process. Regardless, these results confirm that our $\mathrm{B} 3 \mathrm{H}$ assay reliably detects ternary complex formation in the cytoplasm of living E. coli cells.

Reprogramming antigen specificity by genetic selection of a combinatorial library. We next investigated whether the $\mathrm{B} 3 \mathrm{H}$ assay could be used to create designer BsAbs by direct selection of combinatorial libraries. Our objective was to select dual-antigen binders that interacted with a different leucine zipper, namely the leucine zipper domain of $\mathrm{c}$-Jun (JunLZ) ${ }^{15}$, while retaining affinity towards gpD. We first replaced the gene encoding the Gcn4 leucine zipper in pTG-Fv $\mathrm{F}_{2}$-gB with the gene encoding the JunLZ leucine zipper, yielding plasmid $\mathrm{pTJ}-\mathrm{Fv}_{2}-\mathrm{gB}$. Importantly, MC4100A cells transformed with this new plasmid were sensitive to Carb (Fig. 4A). Given that a band corresponding to the full-length $\mathrm{gB}$ chimera was lacking in the periplasm (Fig. 4B), we conclude that no complex between TJ, Fv2, and pG was formed, which would be required to export Bla to the periplasm. Taken together, these results indicate that the scFv-GCN4 domain in $\mathrm{Fv}_{2}$ was specific to $\mathrm{Gcn} 4$ and did not cross-react with JunLZ. Indeed, enzyme-linked immunosorbent assay (ELISA) confirmed that purified $\mathrm{Fv}_{2}$ bound strongly to immobilized Gcn4 but not JunLZ (Fig. 4C).

To reprogram the specificity of the $\mathrm{Fv}_{2}$ from Gcn4 to JunLZ, we randomized three important antigen-binding residues (GLF) in complementarity determining region 3 of the $\mathrm{V}_{\mathrm{H}}$ domain (CDR$\mathrm{H} 3$ ) of scFv-GCN4 using an NNK strategy (Fig. S4A). The scFvGCN4 gene library was cloned in $\mathrm{pTJ}-\mathrm{Fv}_{2}$-gB, resulting in the plasmid library $\mathrm{pTJ}-\mathrm{Fv}_{2}(\mathrm{NNK})$-gB that was used to subsequently transform MC4100A cells. A total of $\sim 8 \times 10^{3}$ library clones were selected on plates containing $20 \mu \mathrm{g} / \mathrm{mL}$ Carb and thirty Carb-resistant clones were picked randomly for further analysis. The plasmids from these positive clones were isolated and used to retransform fresh cells to confirm the growth phenotype. Three of these clones had a clear growth advantage compared to MC4100A cells carrying $\mathrm{pTJ}-\mathrm{Fv}_{2}{ }^{-}$ $\mathrm{gB}$ (Fig. S4B). Sequencing of the CDR-H3 regions of these positive clones revealed three unique amino acid sequences, namely WQL, PAP and TFL (Fig. S4A). We focused on $\mathrm{Fv}_{2}(\mathrm{WQL})$ because this clone conferred the strongest growth advantage to cells (Fig. 4A and Fig. S4B). Immunoblotting revealed that $\mathrm{Fv}_{2}$ (WQL) but not the parental $\mathrm{Fv}_{2}$ was localized in the periplasm when JunLZ was co-expressed as one of the antigens (Fig. 4B). Consistent with the growth phenotypes, only cells expressing $\mathrm{Fv}_{2}(\mathrm{WQL})$ accumulated full-length $\mathrm{gB}$ in the periplasm (Fig. 4B). It should be noted that a smaller $\sim 28-\mathrm{kDa}$ band appeared in the periplasm of control cells (Fig. 4B). We speculate that this band corresponds to a proteolytic product of the $\mathrm{gB}$ chimera that somehow becomes localized in the periplasm. This localization clearly does not involve the hitchhiker mechanism as the $\mathrm{Fv}_{2}$ proteins in these cases are localized exclusively in the cytoplasm. Hence, export of this unidentified fragment must proceed by an undetermined mechanism. The absence of all or most of Bla in this truncation product can be inferred from the fact that the cells producing this truncation product exhibit strong growth inhibition in the presence of Carb. Importantly, our results demonstrate that antigen-binding specificity of one of the $\mathrm{scFv}$ domains could be reprogrammed without compromising the antigen-binding activity of the other. Moreover, the growth advantage and hitchhiker export conferred by $\mathrm{Fv}_{2}(\mathrm{WQL})$ was lost when the target antigen was changed to Gcn 4 (Fig. 4A and B, respectively), indicating that the WQL substitution created a new CDR-H3 that was highly specific for JunLZ. In further support of this exquisite specificity, ELISA experiments revealed strong binding between purified $\mathrm{Fv}_{2}(\mathrm{WQL})$ and JunLZ whereas no measurable binding above background was observed between $\mathrm{Fv}_{2}$ (WQL) and Gcn4 (Fig. 4C).

The equilibrium dissociation constant, $K_{\mathrm{D}}$, for the interaction between $\mathrm{Fv}_{2}$ (WQL) and JunLZ was $6.4 \times 10^{-7} \mathrm{M}$ as determined by Biacore analysis, which was measurably higher than that of the parental $\mathrm{Fv}_{2}$ for Gcn $4\left(K_{\mathrm{D}}=3.1 \times 10^{-8} \mathrm{M}\right)$ (Fig. $4 \mathrm{D}$ and Fig. S5). Nonetheless, isolation of a strong JunLZ binder after just a single round of highly focused mutagenesis (i.e., targeting of only 3 residues in CDR-H3) and selection reveals the potential of the $\mathrm{B} 3 \mathrm{H}$ assay. Moreover, the $K_{\mathrm{D}}$ of $\mathrm{Fv}_{2}(\mathrm{WQL})$ for gpD was $4.2 \times 10^{-5} \mathrm{M}$, which compared favorably to the $K_{\mathrm{D}}$ values measured for the parental $\mathrm{Fv}_{2}$ or scFv-D10 against gpD $\left(8.7 \times 10^{-6} \mathrm{M}\right.$ and $2.2 \times 10^{-5} \mathrm{M}$, respectively) (Fig. $4 \mathrm{D}$ and Fig. S5). Hence, reprogramming $\mathrm{Fv}_{2}$ to bind 


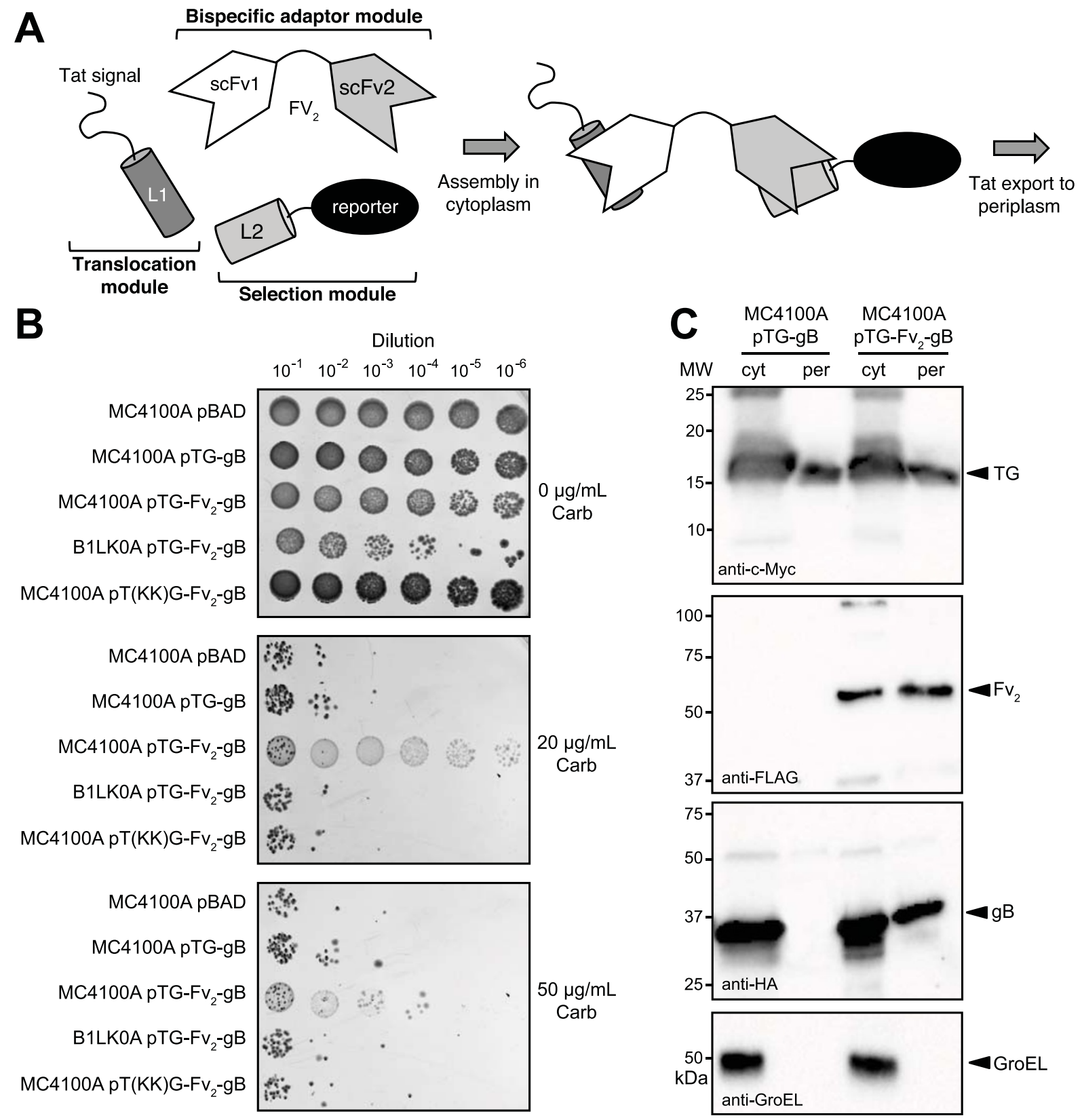

Figure 3 B3H selection system for studying and engineering ternary protein complexes. (A) Schematic of the engineered B3H selection system. The first ligand (L1) modified with a Tat signal peptide serves as the translocation module while the second ligand (L2) fused to the reporter enzyme Bla functions as the selection module. Introduction of a bispecific adaptor module (e.g., bispecific antibody, $\mathrm{Fv}_{2}$ ) creates a physical crosslink between the Tat signal peptide and Bla, thereby resulting in periplasmic accumulation of the ternary complex and conferral of antibiotic resistance to host cells. (B)

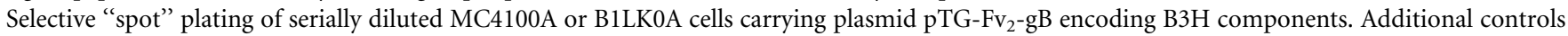
included MC4100A cells carry empty plasmid (pBAD), a plasmid lacking the bispecific $\mathrm{Fv}_{2}$ (pTG-gB), or a plasmid encoding a non-functional KK mutant signal peptide ( $\left.\mathrm{pT}(\mathrm{KK}) \mathrm{G}_{-}-\mathrm{Fv}_{2}-\mathrm{gB}\right)$. Overnight cultures were serially diluted in liquid LB and spot plated on LB-agar supplemented with Carb (0-50 $\mu \mathrm{g} /$ $\mathrm{mL}$ ) and arabinose $(0.2 \% \mathrm{w} / \mathrm{v})$. (C) Western blot analysis of cytoplasmic (cyt) and periplasmic (per) fractions prepared from MC4100A cells carrying pTG-gB or pTG-Fv - gB. Fractions were normalized to an equivalent number of cells prior to loading. Blots were probed with anti-c-Myc, anti-FLAG, or anti-HA antibodies to detect each $\mathrm{B} 3 \mathrm{H}$ component individually. Quality of the fractions was confirmed by probing membranes with an anti-GroEL antibody. Molecular weight (MW) markers are indicated on the left. See Supplementary Fig. S9 for uncropped versions of the images.

JunLZ was accomplished without significantly compromising gpD binding, thereby demonstrating the potential of the $\mathrm{B} 3 \mathrm{H}$ assay for rapid isolation of proteins with dual affinity.

\section{Discussion}

In this study, we uncovered a naturally occurring heterotrimer cotranslocation process involving the aldehyde oxidoreductase PaoABC, which is exported by the Tat translocase as a ternary complex. Specifically, the $\mathrm{PaoB}$ and $\mathrm{PaoC}$ subunits, each lacking a dis- cernable export signal, associate with the signal peptide-containing PaoA subunit to be translocated together across the cytoplasmic membrane. This unique mode of export whereby a polypeptide lacking a signal peptide can be effectively translocated in a piggyback fashion on another polypeptide containing a signal peptide is used by at least one other Tat substrate, namely the E. coli hydrogenase HybOC $^{9}$, as well as by yeast and mammalian peroxisomes, which can translocate homo-oligomeric proteins (thiolase dimers and chloramphenicol acetyl transferase trimers) across their 

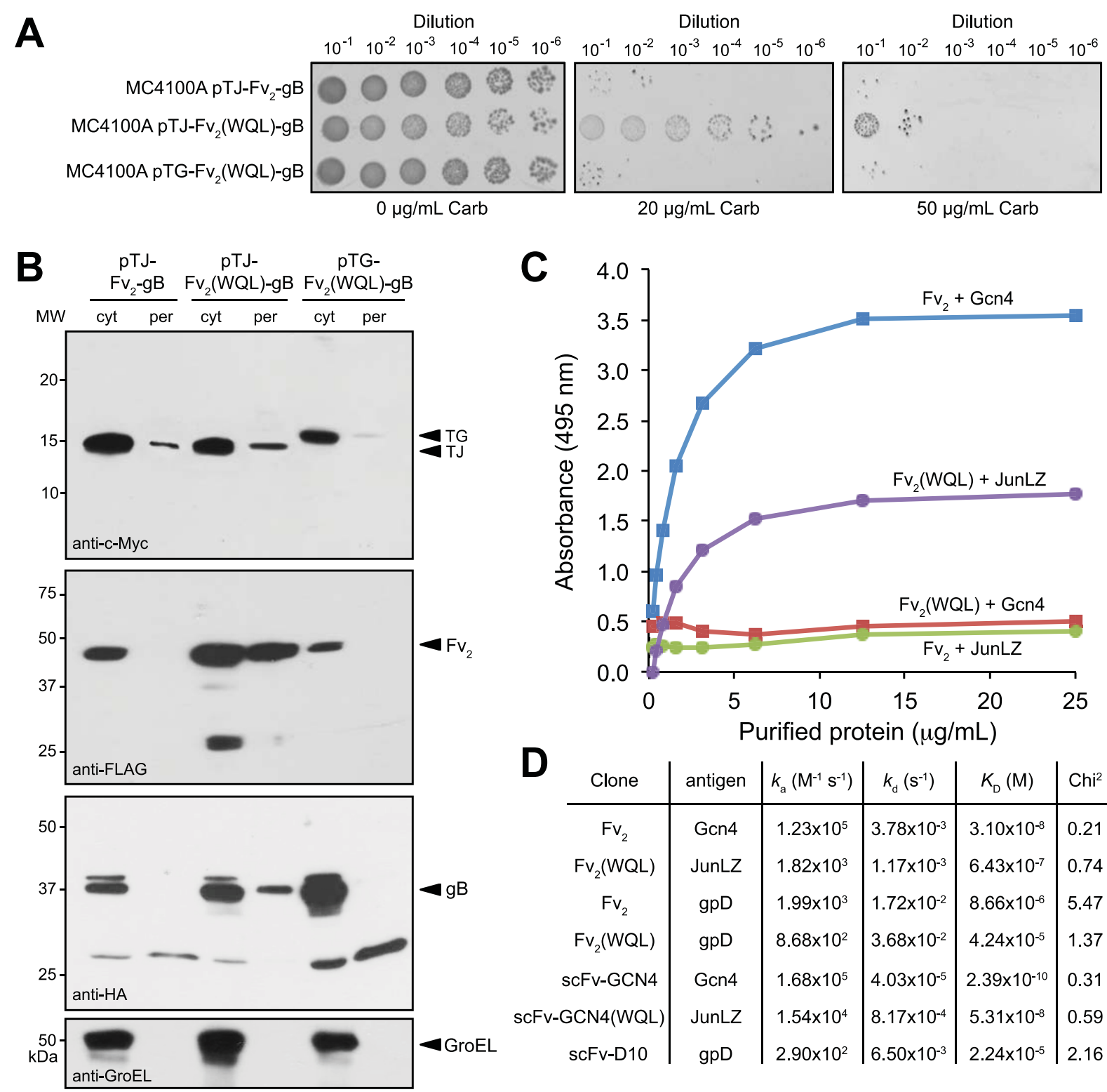

\begin{tabular}{c|c|c|c|c|c} 
Clone & antigen & $k_{\mathrm{a}}\left(\mathrm{M}^{-1} \mathrm{~s}^{-1}\right)$ & $k_{\mathrm{d}}\left(\mathrm{s}^{-1}\right)$ & $K_{\mathrm{D}}(\mathrm{M})$ & $\mathrm{Chi}^{2}$ \\
\hline $\mathrm{Fv}_{2}$ & $\mathrm{Gcn} 4$ & $1.23 \times 10^{5}$ & $3.78 \times 10^{-3}$ & $3.10 \times 10^{-8}$ & 0.21 \\
$\mathrm{Fv}_{2}(\mathrm{WQL})$ & $\mathrm{JunLZ}$ & $1.82 \times 10^{3}$ & $1.17 \times 10^{-3}$ & $6.43 \times 10^{-7}$ & 0.74 \\
$\mathrm{Fv}_{2}$ & $\mathrm{gpD}$ & $1.99 \times 10^{3}$ & $1.72 \times 10^{-2}$ & $8.66 \times 10^{-6}$ & 5.47 \\
$\mathrm{Fv}_{2}(\mathrm{WQL})$ & $\mathrm{gpD}$ & $8.68 \times 10^{2}$ & $3.68 \times 10^{-2}$ & $4.24 \times 10^{-5}$ & 1.37 \\
$\mathrm{scFv}-\mathrm{GCN} 4$ & $\mathrm{Gcn} 4$ & $1.68 \times 10^{5}$ & $4.03 \times 10^{-5}$ & $2.39 \times 10^{-10}$ & 0.31 \\
scFv-GCN4(WQL) & $\mathrm{JunLZ}$ & $1.54 \times 10^{4}$ & $8.17 \times 10^{-4}$ & $5.31 \times 10^{-8}$ & 0.59 \\
scFv-D10 & $\mathrm{gpD}$ & $2.90 \times 10^{2}$ & $6.50 \times 10^{-3}$ & $2.24 \times 10^{-5}$ & 2.16
\end{tabular}

Figure $4 \mid$ Reprogramming antigen specificity using B3H-mediated library selection. (A) Selective spot plating of serially diluted MC4100A cells carrying pTJ-Fv $-\mathrm{gB}, \mathrm{pTJ}-\mathrm{Fv}_{2}(\mathrm{WQL})$-gB, or pTG-Fv 2 (WQL)-gB. Overnight cultures were serially diluted in liquid LB and spot plated on LB-agar supplemented with Carb $(0-50 \mu \mathrm{g} / \mathrm{mL})$ and arabinose $(0.2 \% \mathrm{w} / \mathrm{v})$. (B) Western blot analysis of cytoplasmic (cyt) and periplasmic (per) fractions prepared from the same cells as in (A). Fractions were normalized to an equivalent number of cells prior to loading. Blots were probed with anti-c-Myc, anti-FLAG, and anti-HA antibodies to detect each component individually. Quality of fractions was confirmed by probing membranes with an antiGroEL antibody. Molecular weight (MW) markers are indicated on the left. See Supplementary Fig. S10 for uncropped versions of the images. (C) Binding activity of purified $\mathrm{Fv}_{2}$ and $\mathrm{Fv}_{2}$ (WQL) proteins against immobilized MBP-GCN4 and MBP-JunLZ antigens as measured by ELISA. Bound Fv 2 molecules were detected with anti-His antibodies. (D) Affinity determination of purified bispecific $\mathrm{Fv}_{2} \mathrm{~S}$ or unfused scFvs to cognate antigens as measured by surface plasmon resonance (SPR). Cognate antigens were immobilized on $\mathrm{CM} 5$ chips and the response of varied amounts of bispecific $\mathrm{Fv}_{2} \mathrm{~S}$ or unfused scFvs was compared with an empty flow cell. The binding kinetics of bispecific $\mathrm{Fv}_{2} \mathrm{~s}$ or unfused scFvs were monitored using Biacore and affinity values were obtained by fitting the equilibrium binding responses with a 1:1 Langmuir binding model using a simultaneous non-linear program (for sensorgrams, see Fig. S5).

membranes ${ }^{28}$. In E. coli, at least five additional Tat substrates (out of the 27 proteins in E. coli known or predicted to bear N-terminal Tat signal peptides) may also be translocated as heterodimers: DmsAB, FdnGH, FdoGH, HyaAB, YnfEG, and $\mathrm{YnfFG}^{10}$. Some of these (HybO, HyaA, FdnH and FdoH) are components of larger multisubunit hydrogenase or formate dehydrogenase respiratory complexes that exhibit a heterotrimeric $\alpha \beta \gamma$ structure ${ }^{29}$. However, in these examples, only the $\alpha \beta$ complex (e.g., FdnGH) is predicted to be exported as a preformed unit by a Tat signal peptide located on only one of the subunits; the $\gamma$-subunit (e.g., FdnI) is a signal recognition particle (SRP)-dependent integral membrane protein. Thus, to our knowledge, PaoABC is the only $\alpha \beta \gamma$ heterotrimer identified so far whose complete export depends on the Tat pathway. And while no upper limit has been determined for the size of a molecule that can be handled by the Tat translocase, PaoABC represents one of the largest structures $(135-\mathrm{kDa})$ exported to date.

The translocation of PaoABC, like its HybOC counterpart, appears to involve an interesting QC phenomenon whereby absence of any one of the subunits prevents export of the others. The fact that the signal peptide itself is necessary but not sufficient for 
translocation suggests the involvement of an accessory factor, such as a dedicated molecular chaperone, that helps to coordinate PaoABC maturation (i.e., cofactor insertion, subunit assembly) and prevent wasteful export of misfolded or immature enzymes. Indeed, such dedicated 'proofreading' chaperones have been identified for many Tat substrates, including $\alpha \beta$ heterodimers whose export follows the hitchhiker mechanism ${ }^{10}$. In the case of HybOC, the HybE chaperone binds to the Tat signal peptide of HybO, probably acting to mask the signal from the Tat translocase while simultaneously preventing premature folding during cofactor loading ${ }^{30}$. Likewise, $\mathrm{PaoD}$, is predicted to fulfill this role for PaoABC by participating in the modification and insertion of the Moco cofactor and coordinating these events with oligomer export ${ }^{17}$. In addition to dedicated chaperones, it is also plausible that the folding and assembly state of PaoABC may be monitored at a later stage in the export process. For example, the Tat translocase itself appears to have an inbuilt ability to discriminate between folded and mis/unfolded substrate proteins, allowing export of only the former ${ }^{2,13}$.

The interdependence of each subunit for export also implies direct contact between the subunits and favors a model in which the formation of a complex precedes translocation. In support of this model, we obtained several lines of evidence that $\mathrm{PaoA}, \mathrm{PaoB}$, and $\mathrm{PaoC}$ form a stable $\alpha \beta \gamma$ heterotrimer in vivo, in agreement with previous studies $^{17,18}$. Interestingly, our BN-PAGE analysis revealed the formation of a possible $(\alpha \beta \gamma)_{2}$ dimer of PaoABC trimers, which could also be seen as a very minor product in the gel filtration profile. While further experiments are needed to unequivocally establish $(\alpha \beta \gamma)_{2}$ complex formation, such a complex is not entirely unexpected in light of other molybdoenzymes in the xanthine oxidase (XO) family, of which $\mathrm{PaoABC}$ is a member. For example, the xanthine dehydrogenase $(\mathrm{XDH})$ system in Rhodobacter capsulatus exists as a noncovalent $(\alpha \beta)_{2}$ dimer of heterodimers ${ }^{31}$, where the $\alpha$ subunit XdhA is homologous to the PaoAB complex and the $\beta$ subunit $\mathrm{XdhB}$ is homologous to PaoC.

From a technological standpoint, a notable outcome of the studies described here was the creation of a $\mathrm{B} 3 \mathrm{H}$ selection system for studying and engineering ternary protein complexes. We demonstrated that ternary protein interactions could be readily detected and optimized simply by selective plating of bacterial cells, a process that obviates the need for purification or immobilization of either binding target. In particular, we showed that the $\mathrm{B} 3 \mathrm{H}$ selection was compatible with BsAbs (e.g., bispecific $\mathrm{Fv}_{2}$ ), which have great potential as biotherapeutics due to their dual target recognition enabling simultaneous inhibition of multiple cell surface receptors, cross-linking of two receptors, and recruitment of effector cells ${ }^{32-34}$. Because most $\mathrm{BsAb}$ formats, in particular tandem scFvs, are relatively small, aglycosylated proteins, their production is ideally suited for a simple, cost-effective host organism like E. coli. However, attempts to produce functional tandem scFvs in $E$. coli have been largely unsuccessful $^{35-38}$, prompting a reliance upon eukaryotic host cells for improved functional expression ${ }^{35}$. Moreover, due to the lack of available techniques for selecting binders against two antigens simultaneously, BsAbs are typically isolated for their affinity to each antigen independently. This can be problematic because molecular recombination of two pre-existing, high-affinity binding proteins may yield a non-functional BsAb due to steric hindrance issues upon binding antigen or misfolding of the bispecific protein itself.

Notably, the $\mathrm{B} 3 \mathrm{H}$ technology described here has the potential to overcome these challenges. First, simultaneous interaction between the dual-affinity BsAb and its two cognate antigens is required to confer selective growth. Second, the intrinsic folding QC of the Tat translocase $\mathrm{e}^{2,13}$, which only exports properly folded, nonaggregating proteins, should favor the isolation of stable and soluble BsAbs. While not demonstrated here, we predict that our $\mathrm{B} 3 \mathrm{H}$ selection system could be used to select for trans-acting factors (e.g., molecular chaperones) that promote folding, solubility and/or stability of
BsAbs, such as E. coli $\mathrm{FkpA}^{39}$. Finally, in addition to BsAb development, we envision that the $\mathrm{B} 3 \mathrm{H}$ technology could easily be leveraged for the creation of bifunctional adaptor proteins that (i) bring together two activities for rewiring cellular signaling or colocalizing metabolic pathways $s^{40,41}$, (ii) support orthogonal affinity purification strategies using two different matrices ${ }^{42}$, and (iii) function as immunoprobes in diagnostic assays ${ }^{34}$.

Finally, it should be pointed out that a small handful of threehybrid assays in yeast and bacteria have been reported ${ }^{43-47}$. In every case, the assays rely on the reconstitution of a transcriptional activator that subsequently turns on expression of a reporter gene. This assay format has proven to be prone to a high frequency of false positives that arise from spurious transcriptional activation ${ }^{48}$, with $>50 \%$ of the data generated using a transcription-based format likely to be false positives ${ }^{49}$. Because our assay does not involve DNA binding, it is not susceptible to spurious transcriptional activation. Another advantage of our $\mathrm{B} 3 \mathrm{H}$ approach is that the Tat system only exports correctly folded, non-aggregated proteins and protein complexes $^{13,15}$, thereby providing a built-in safeguard against false positives arising from the interaction of misfolded proteins. Also, because the assay requires the complex to remain associated during membrane transport, it stands to reason that relatively weak interactions will not be selected, which is especially advantageous for antibody development applications such as described here.

\section{Methods}

Bacterial strains and growth conditions. All strains used in this study are given in Supplementary Table S1. Parental strain E. coli BW25113 ${ }^{50}$ and isogenic Keio derivatives JW0280 (BW25113 $\Delta p a o A:: K a n), ~ J W 0279$ (BW25113 $\Delta p a o B:: K a n$ ), and JW0278 (BW25113 $\Delta$ paoC::Kan) ${ }^{51}$ were used for PaoABC expression experiments. To eliminate the possibility of interference from endogenous Pao proteins, a triple knockout strain was prepared by sequential knockout of paoB and paoC in JW0280. This involved transformation of JW0280 with plasmid pCP20 to excise the Kan resistance gene in $p a o A$ as described ${ }^{50}$. The resulting strain was then subjected to $\mathrm{P} 1$ transduction using JW0279 ( $\triangle p a o B::$ Kan $)$ as donor strain, followed by removal of the Kan marker in paoB using pCP20. Finally, the paoC gene was knocked out by P1 transduction using JW0278 as donor strain, resulting in strain HL0281 (BW25113 $\triangle$ paoAB-paoC::Kan). Wild-type E. coli $\mathrm{MC} 4100$ and its isogenic $\Delta$ tat $C$ derivative called B1LK0 ${ }^{22}$ were used for some PaoABC experiments. MC4100A (MC4100 ara $^{+}$) and B1LK0A (B1LK0 $\left.\mathrm{ara}^{+}\right)^{52}$ were used for all BH3 experiments. E. coli JM109 was used for cloning genes while E. coli BL21(DE3) (Novagen) and SHuffle T7 Express (New England Biolabs) were used for cytoplasmic expression and purification of MBP-Gcn4, MBP-JunLZ, and all $\mathrm{Fv}_{2} / \mathrm{scFv}$ constructs. Typically, cultures were grown in Luria-Bertani (LB) medium supplemented with the appropriate antibiotic, and protein expression was induced when the cells reached $\mathrm{OD}_{600} \approx 0.4-0.6$ with isopropyl $\beta$-D-1-thiogalactopyranoside (IPTG; $0.1-0.5 \mathrm{mM})$ or arabinose $(0.2 \% \mathrm{w} / \mathrm{v})$ depending on the plasmid used. Antibiotics were provided at the following concentrations: chloramphenicol $(\mathrm{Cm}), 20 \mu \mathrm{g} / \mathrm{mL}$; kanamycin (Kan), $50 \mu \mathrm{g} / \mathrm{mL}$; ampicillin (Amp), $100 \mu \mathrm{g} / \mathrm{mL}$; Carb, $0-1,000 \mu \mathrm{g} / \mathrm{mL}$ for selection.

Plasmid construction. All plasmids used in this study are given in Supplementary Table S1.

To generate a synthetic paoABC operon, amplicon 1 (vector region-RBS-PaoA-c $\mathrm{Myc}$ ), amplicon 2 (c-myc-RBS-PaoB-FLAG), and amplicon 3 (FLAG-RBS-PaoCHA-stop-vector region) were PCR amplified from E. coli genomic DNA using primers that appended overlapping regions (Fig. S1A). The resulting three PCR products and linearized pTrc99Y were joined in vivo by the yeast lazy-bone assembly method $^{53}$, resulting in plasmid pPaoABC (Fig. S1B). Omission of each one of the pao genes in subsequent gene manipulations yielded plasmids $\mathrm{pPao}(\triangle \mathrm{A}) \mathrm{BC}$,

pPaoA $(\Delta \mathrm{B}) \mathrm{C}$, and $\mathrm{pPaoAB}(\Delta \mathrm{C})$. The plasmid $\mathrm{pPaoAhBC}$, encoding a $6 \mathrm{xHis}$-tagged version of $\mathrm{PaoA}$, was generated from $\mathrm{PPaoABC}$ by exchanging the DNA between $\mathrm{XbaI} /$ NotI encoding PaoA with a DNA fragment encoding PaoA-6xHis. To generate the $\mathrm{B} 3 \mathrm{H}$ selection plasmid, amplicon $\mathrm{B} 1$ (vector region-RBS-ssTorA-Gcn4-c-Myc), amplicon B2 (c-Myc-RBS-scFv-GCN4-GS linker), amplicon B3 (GS linker-scFvD10-FLAG-RBS-N-terminal gpD), amplicon B4 (N-terminal gpD-HA), and amplicon B5 (HA-mature Bla-vector region) were PCR amplified from laboratory stock plasmid $\mathrm{DNA}^{15,27}$ using primers that appended overlapping regions (Fig. S3A). The resulting five PCR products and linearized pBAD18-Cm were assembled using Gibson assembly (New England Biolabs) according to the manufacturer's instructions, resulting in plasmid pTG-Fv $v_{2}$ gB (Fig. 3B). Plasmid pTG-gB was constructed from $\mathrm{pTG}-\mathrm{Fv}_{2}-\mathrm{gB}$ by omitting the tandem $\mathrm{scFv}$ portion in subsequent gene manipulations. Plasmid $\mathrm{pT}(\mathrm{KK}) \mathrm{G}-\mathrm{Fv}_{2}-\mathrm{gB}$ was generated using a QuikChange site-directed mutagenesis kit (Agilent) with two antisense primers (forward: $5^{\prime}$-cga tct ctt tca ggc atc aaa gaa gcg ttt tct ggc aca act cgg cgg- $3^{\prime}$; reverse: $5^{\prime}$ - ccg ccg agt tgt gcc aga aaa cgc $\mathrm{ttc} t \mathrm{tt}$ gat gcc tga aag aga tcg- $\left.3^{\prime}\right)$. Plasmid $\mathrm{pTJ}-\mathrm{Fv}_{2}-\mathrm{gB}$ was generated from $\mathrm{pTG}-\mathrm{Fv}_{2}-\mathrm{gB}$ by first exchanging Gcn 4 with JunLZ and then transferring the entire insert into 
pBAD18-Cm. For expression and purification of the target antigens Gcn 4 and JunLZ, plasmid pET28-MBP-TEV was constructed by PCR amplifying the DNA encoding $E$. coli maltose-binding protein (MBP) with a C-terminal TEV cleavage site added by primer extension. The resulting PCR product was ligated between NdeI/BamHI sites of pET28a(+) (Novagen). Plasmids pET28-MBP-Gcn4 and pET28-MBP-JunLZ were generated by inserting PCR-amplified Gcn4 and JunLZ into BamHI/HindIII restriction sites of pET28-MBP-TEV. For expression and purification of gpD, plasmid pET28-gpD was constructed by double ligation of dimerizing primers encoding a 6xHis tag and HA tag with $5^{\prime} \mathrm{NcoI}$ and $3^{\prime}$ NdeI overhangs and PCR-amplified gpD with $5^{\prime} \mathrm{NdeI}$ and $3^{\prime}$ HindIII restriction sites into pET28a $(+)$ between NcoI/HindIII restriction sites. For cytoplasmic expression and purification of $\mathrm{Fv}_{2}$ and scFv proteins, recombinant antibody genes were PCR amplified from laboratory stock plasmid DNA $^{15,27}$ during which a C-terminal FLAG tag was introduced by using primer extension. The resulting PCR products were then ligated into pET28a $(+)$ between $\mathrm{NcoI} / \mathrm{HindIII}$ restriction sites without a stop codon in order to include the C-terminal 6xHis-tag in pET28a $(+)$. This yielded pET28-scFv-GCN4, pET28-scFvGCN4(WQL), pET28-scFv-D10, pET28-Fv 2 and pET28-Fv 2 (WQL), where each included dual C-terminal FLAG/6xHis tags. All plasmids were confirmed by DNA sequencing.

Library construction. Random mutagenesis using degenerate (NNK) forward primers and degenerate (MNN) reverse primers was used to create a 3-residue library containing mutations in the first three amino acids of the CDR-H3 (XXXDY) of the scFv-GCN4 domain of $\mathrm{Fv}_{2}$. Random forward primers were encoded by the sequence $5^{\prime}$-gat act gct ctc tat tac tgc gtg aca NNK NNK NNK gac tac tgg ggg cag ggc acg ctg gtt $\mathrm{g}-3^{\prime}$, where $\mathrm{N}$ is an equimolar mixture of all four nucleotides and $\mathrm{K}$ is an equimolar mixture of $\mathrm{G}$ and $\mathrm{T}$, and random reverse primers were encoded by the sequence $5^{\prime}$ caa cca gcg tgc cct gcc ccc agt agt cMN NMN NMN Ntg tca cgc agt aat aga gag cag tat $c-3^{\prime}$, where $\mathrm{M}$ is an equimolar mixture of $\mathrm{A}$ and $\mathrm{C}$ and $\mathrm{N}$ is an equimolar mixture of all four nucleotides. These primers were used to generate a mutant library from plasmid pTG- $\mathrm{Fv}_{2}-\mathrm{gB}$ as template according to the QuikChange mutagenesis protocol (Agilent). After 18 cycles of amplification, randomized scFv-GCN4(XXX) sequences were amplified with forward primer ( $5^{\prime}$ - ggt cgc tag cat gcg aga tat cgt tat gac $\left.\mathrm{c}-3^{\prime}\right)$ and reverse primer $\left(5^{\prime}\right.$-tca agg tcg acc gat $\mathrm{ccg}$ cca cc- $\left.3^{\prime}\right)$. The resulting PCR products, encoding a DNA library of scFv-GCN4(XXX) sequences, were cloned into pTJ- $\mathrm{Fv}_{2}$ $\mathrm{gB}$ using NheI and SalI restriction enzymes. The resulting plasmid was used to transform E. coli MC4100 and then selected on LB agar containing Cm to recover clones containing library plasmids. Library cells were pooled and their plasmids were isolated for selection experiments.

Selective growth assays and library selection. For $\mathrm{B} 3 \mathrm{H}$ experiments, cells carrying pTG- $\mathrm{Fv}_{2}-\mathrm{gB}$ plasmid or equivalent controls were grown overnight in LB containing $20 \mu \mathrm{g} / \mathrm{mL} \mathrm{Cm}$. Screening of cells was performed by spreading an equivalent number of serially diluted overnight cells directly onto LB agar plates supplemented with $0.2 \%$ $(\mathrm{w} / \mathrm{v})$ arabinose and 0,20 or $50 \mu \mathrm{g} / \mathrm{mL}$ Carb and incubating at $30^{\circ} \mathrm{C}$ for $24 \mathrm{~h}$. Library selections were performed by electroporating the tricistronic library plasmid pTJ$\mathrm{Fv}_{2}(\mathrm{NNK})$-gB into E. coli MC4100 cells followed by direct plating on LB agar supplemented with $0.2 \%(\mathrm{w} / \mathrm{v})$ arabinose and $10 \mu \mathrm{g} / \mathrm{mL}$ Carb. Thirty randomly chosen Carb-resistant clones were subjected to further characterization by spreading an equivalent number of serially diluted overnight cells directly onto LB agar plates supplemented with $0.2 \%$ (w/v) arabinose and $10 \mu \mathrm{g} / \mathrm{mL}$ Carb and incubating at $30^{\circ} \mathrm{C}$ for $48 \mathrm{~h}$. Additionally, these thirty clones were subjected to sequencing to eliminate false positives from direct recombination of the TorA-derived signal peptide with Bla. Finally, selected cells were confirmed by spot plating $5 \mu \mathrm{L}$ of overnight cells that had been normalized in fresh $\mathrm{LB}$ to $\mathrm{OD}_{600} \approx 1$ onto $\mathrm{LB}$ agar plates supplemented with $0.2 \%(\mathrm{w} / \mathrm{v})$ arabinose and $0-50 \mu \mathrm{g} / \mathrm{mL}$ Carb and incubating at $30^{\circ} \mathrm{C}$ for $48 \mathrm{~h}$.

Subcellular fractionation and Western blot analysis. The subcellular fractionation was performed as described elsewhere ${ }^{54}$. Briefly, to prepare subcellular fractions for Western blot analysis, we pelleted and washed $15 \mathrm{~mL}$ of induced culture with subcellular fractionation buffer ( $30 \mathrm{mM}$ Tris- $\mathrm{HCl}, 1 \mathrm{mM}$ EDTA, and $0.6 \mathrm{M}$ sucrose). Cells were resuspended in $1 \mathrm{~mL}$ subcellular fractionation buffer and then incubated for $10 \mathrm{~min}$ at room temperature and spun down. After addition of $266 \mu \mathrm{L}$ of $5 \mathrm{mM} \mathrm{MgSO}_{4}$, cells were incubated for $10 \mathrm{~min}$ on ice. Cells were spun down, and the supernatant was taken as the periplasmic fraction. The pellet was treated with BugBuster Master Mix (Novagen) for $20 \mathrm{~min}$ at room temperature. Following centrifugation at 12,000 rpm at room temperature for $5 \mathrm{~min}$, the second supernatant was taken as the soluble cytoplasmic fraction, and the pellet was retained as the insoluble fraction. Soluble lysates were normalized using a total protein assay (BioRad) with BSA as standard and samples were normalized to load $20 \mu \mathrm{g}$ total protein per lane. Cytoplasmic and periplasmic proteins were separated by SDS-PAGE and Western blotted as previously described ${ }^{13}$. The following primary antibodies were used: rabbit anti-c-Myc clone 9E10 (1:5,000; Sigma); rabbit anti-HA (1:1500; Abcam); rabbit anti-GroEL ( $1: 20,000$; Sigma). Secondary anti-rabbit polyclonal antibodies conjugated to horseradish peroxidase (HRP) $(1: 2500$; Promega) were used for detection. Also, mouse anti-FLAG-HRP conjugated to HRP $(1: 3,000$; Sigma) and rabbit anti-His antibodies conjugated to HRP $(1: 10,000$; Abcam) were used depending on affinity tags used.

Protein purification. Gcn4 and JunLZ were expressed as MBP fusions from pET28MBP-Gcn4 and pET-MBP-JunLZ, respectively, in the cytoplasm of E. coli BL21(DE3) cells. The resulting MBP fusions were each purified using amylose affinity chromatography. Briefly, soluble lysates were applied to amylose columns, and the columns were washed with five times the bead volume with a buffer containing $1 \mathrm{mM}$ EDTA, $200 \mathrm{mM} \mathrm{NaCl}, 20 \mathrm{mM}$ Tris/Cl (pH 7.4). Fusion proteins were eluted using a buffer containing $1 \mathrm{mM}$ EDTA, $200 \mathrm{mM} \mathrm{NaCl}, 10 \mathrm{mM}$ maltose, $20 \mathrm{mM} \mathrm{Tris} / \mathrm{Cl}$ (pH 7.4). After purification, eluted MBP fusions were further desalted using a $\mathrm{Zeba}^{\mathrm{TM}}$ Spin Desalting Column (Fisher) into $20 \mathrm{mM}$ Tris/Cl(pH.4). The gpD and scFv-D10 proteins were expressed from $\mathrm{pET} 28$-gpD and pET28-scFv-D10, respectively, in BL21(DE3) cells, while scFv-GCN4 and scFv-GCN4(WQL) were expressed in the cytoplasm of SHuffle T7 Express cells (New England Biolabs) from pET28-scFvGCN4 and pET28-GCN4(WQL), respectively. All of these 6xHis-tagged proteins were purified using Ni-NTA HisTrap FPLC columns (GE Healthcare, ÄKTA) according to manufacturer's protocols. Briefly, soluble lysates were applied to NiNTA columns, and the columns were washed with five times the column volume with a buffer containing $50 \mathrm{mM} \mathrm{NaH}_{2} \mathrm{PO}_{4}, 300 \mathrm{mM}$ sodium chloride, and $20 \mathrm{mM}$ imidazole ( $\mathrm{pH} 8.0$ ). The proteins were all eluted using a buffer containing $50 \mathrm{mM}$ $\mathrm{NaH}_{2} \mathrm{PO}_{4}, 300 \mathrm{mM}$ sodium chloride, and $250 \mathrm{mM}$ imidazole ( $\mathrm{pH} 8.0$ ). Following FPLC purification, eluted proteins were further desalted as described above. The bispecific proteins $\mathrm{Fv}_{2}$ and $\mathrm{Fv}_{2}(\mathrm{WQL})$ were expressed in the cytoplasm of SHuffle T7 Express cells from pET28- $\mathrm{Fv}_{2}$ and $\mathrm{pET} 28-\mathrm{Fv}_{2}(\mathrm{WQL})$, respectively. After cell lysis with Bugbuster ${ }^{\mathrm{TM}}$ (Novagen), cell debris was partially removed by centrifugation at $6000 \times$ g. The resulting turbid supernatant was separated into soluble and insoluble fractions by centrifugation at $30,000 \times \mathrm{g}$. To improve the recovery yield of the bispecifics without affecting folding, we resolubilized the insoluble fraction with $0.5 \%$ sarkosyl ${ }^{55}$. Resolubilized material was then mixed with the soluble fraction in HisTrap binding buffer to a final concentration of $0.1 \%$ sarkosyl. Also, Triton X-100 and CHAPS was added to these samples to a final concentration of $1 \%$ and $10 \mathrm{mM}$, respectively, to improve protein binding to the Ni-NTA resin ${ }^{55}$. The resulting soluble 6xHis-tagged $\mathrm{Fv}_{2}$ and $\mathrm{Fv}_{2}$ (WQL) proteins were purified and desalted as described above. Final purity of all proteins was confirmed by SDS-PAGE (Fig. S6).

Biochemical analysis of PaoABC complex formation. PaoA with a 6xHis-tag was co-expressed with PaoB-FLAG and PaoC-HA from plasmid pPaoAhBC and soluble lysates were prepared as described in the Materials and Methods. The 6xHis-tagged $\mathrm{PaoA}$ and associated proteins was purified using Ni-NTA protein purification spin columns (Qiagen) according to manufacturer's protocols. Briefly, soluble lysates were applied to the columns, and the columns were washed four times with a buffer containing $50 \mathrm{mM} \mathrm{NaH}{ }_{2} \mathrm{PO}_{4}, 300 \mathrm{mM}$ sodium chloride, and $20 \mathrm{mM}$ imidazole ( $\mathrm{pH}$ 8.0). Associated proteins were eluted using a buffer containing $50 \mathrm{mM} \mathrm{NaH} \mathrm{PO}_{4}$, $300 \mathrm{mM}$ sodium chloride, and $500 \mathrm{mM}$ imidazole ( $\mathrm{pH}$ 8.0). After spin-column purification, eluted samples were further desalted using a Zeba ${ }^{\mathrm{TM}}$ Spin Desalting Column (Thermo Scientific) into $20 \mathrm{mM}$ Tris/Cl buffer (pH 7.4). Purified samples were then analyzed by SDS-PAGE and immunoblotting. Separation by BN-PAGE (Novex NativePAGE, Life Technologies) was performed as previously described ${ }^{56}$ and according to the manufacturer's protocols. Molecular weights on BN-PAGE were estimated by RF value using a native marker as standard.

Enzyme-linked immunosorbent assay. Enzyme-linked immunosorbent assay (ELISA) was used to evaluate the binding of purified $\mathrm{Fv}_{2}$ proteins to $\mathrm{Gcn} 4$ and JunLZ ELISA plates were coated overnight at $4^{\circ} \mathrm{C}$ with $50 \mu \mathrm{L} /$ well of each antigen in PBS $(10 \mu \mathrm{g} / \mathrm{mL})$. Plates were then blocked at room temperature for $2 \mathrm{~h}$ with $2 \%$ nonfat milk in PBS. After washing plates with PBS supplemented with $0.1 \%$ Tween 20 (PBST), purified protein samples serially diluted in PBS with $50 \mu \mathrm{g} / \mathrm{mL}$ BSA (PBSBSA) were added to the plates $(50 \mu \mathrm{L} /$ well). Plates were incubated for $1 \mathrm{~h}$ at room temperature and then washed with PBST. HRP-conjugated anti-His antibody ( $1: 10,000$; Abcam) in PBS-BSA was added to the plates (50 $\mu \mathrm{L} /$ well). After $1 \mathrm{~h}$ of incubation at room temperature, plates were washed and then incubated with SigmaFast OPD HRP substrate (Sigma) for $20 \mathrm{~min}$. The reaction was quenched with $3 \mathrm{~N} \mathrm{H}_{2} \mathrm{SO}_{4}$, and the absorbance of the wells was measured at $490 \mathrm{~nm}$.

Surface plasmon resonance. Surface plasmon resonance (SPR) experiments were performed using a Biacore 3000 biosensor (GE Healthcare). Purified MBP-Gcn4, MBP-JunLZ and gpD were immobilized on CM5 sensor chips at $10 \mu \mathrm{g} / \mathrm{ml}$ using an amine coupling kit at $\mathrm{pH} 4.0$ until reaching $1200 \mathrm{RU}, 1000 \mathrm{RU}$, and $260 \mathrm{RU}$, respectively, according to the manufacturer's instructions. The binding analyses were carried out in HBS-EP Buffer (GE Healthcare Life Sciences) at a flow rate of $30 \mu \mathrm{l} /$ min. Each $\mathrm{Fv}_{2}$ or scFv protein was associated with ligands for $3 \mathrm{~min}$, and then dissociation phases were observed. During SPR experiments, flow cells were regenerated by injection of $10 \mathrm{mM}$ glycine ( $\mathrm{pH}$ 2.0) followed by thorough washing with the running buffer. Additionally, a blank cell on the same sensor chip was used as a reference to correct for non-specific binding. Kinetic parameters were calculated using BIAevaluation software 3.2. All sensorgrams were fitted to a 1:1 Langmuir binding model using a simultaneous non-linear program.

1. Palmer, T. \& Berks, B. C. The twin-arginine translocation (Tat) protein export pathway. Nat Rev Microbiol 10, 483-496 (2012).

2. Rocco, M. A., Waraho-Zhmayev, D. \& DeLisa, M. P. Twin-arginine translocase mutations that suppress folding quality control and permit export of misfolded substrate proteins. Proc Natl Acad Sci U S A 109, 13392-13397 (2012).

3. Bernhardt, T. G. \& de Boer, P. A. The Escherichia coli amidase AmiC is a periplasmic septal ring component exported via the twin-arginine transport pathway. Mol Microbiol 48, 1171-1182 (2003). 
4. Ize, B., Stanley, N. R., Buchanan, G. \& Palmer, T. Role of the Escherichia coli Tat pathway in outer membrane integrity. Mol Microbiol 48, 1183-1193 (2003).

5. Halbig, D., Wiegert, T., Blaudeck, N., Freudl, R. \& Sprenger, G. A. The efficient export of NADP-containing glucose-fructose oxidoreductase to the periplasm of Zymomonas mobilis depends both on an intact twin-arginine motif in the signal peptide and on the generation of a structural export signal induced by cofactor binding. Eur J Biochem 263, 543-551 (1999).

6. Gralnick, J. A., Vali, H., Lies, D. P. \& Newman, D. K. Extracellular respiration of dimethyl sulfoxide by Shewanella oneidensis strain MR-1. Proc Natl Acad Sci U S A 103, 4669-4674 (2006)

7. Rondelet, A. \& Condemine, G. SurA is involved in the targeting to the outer membrane of a Tat signal sequence anchored protein. J Bacteriol 194, 6131-6142 (2012).

8. Hatzixanthis, K., Palmer, T. \& Sargent, F. A subset of bacterial inner membrane proteins integrated by the twin-arginine translocase. Mol Microbiol 49, 1377-1390 (2003)

9. Rodrigue, A., Chanal, A., Beck, K., Muller, M. \& Wu, L. F. Co-translocation of a periplasmic enzyme complex by a hitchhiker mechanism through the bacterial tat pathway. J Biol Chem 274, 13223-13228 (1999).

10. Palmer, T., Sargent, F. \& Berks, B. C. Export of complex cofactor-containing proteins by the bacterial Tat pathway. Trends Microbiol 13, 175-180 (2005).

11. James, M. J., Coulthurst, S. J., Palmer, T. \& Sargent, F. Signal peptide etiquette during assembly of a complex respiratory enzyme. Mol Microbiol 90, 400-414 (2013)

12. Leu, W. M., Chen, L. Y., Liaw, L. L. \& Lee, Y. H. Secretion of the Streptomyces tyrosinase is mediated through its trans-activator protein, MelC1. J Biol Chem 267, 20108-20113 (1992).

13. DeLisa, M. P., Tullman, D. \& Georgiou, G. Folding quality control in the export of proteins by the bacterial twin-arginine translocation pathway. Proc Natl Acad Sci U S A 100, 6115-6120 (2003).

14. Strauch, E. M. \& Georgiou, G. A bacterial two-hybrid system based on the twinarginine transporter pathway of E. coli. Protein Sci 16, 1001-1008 (2007).

15. Waraho, D. \& DeLisa, M. P. Versatile selection technology for intracellular protein-protein interactions mediated by a unique bacterial hitchhiker transport mechanism. Proc Natl Acad Sci U S A 106, 3692-3697 (2009).

16. Waraho, D. \& DeLisa, M. P. Identifying and optimizing intracellular protein protein interactions using bacterial genetic selection. Methods Mol Biol 813, 125-143 (2012).

17. Neumann, M. et al. A periplasmic aldehyde oxidoreductase represents the first molybdopterin cytosine dinucleotide cofactor containing molybdo-flavoenzyme from Escherichia coli. FEBS J 276, 2762-2774 (2009).

18. Otrelo-Cardoso, A. R. et al. Structural data on the periplasmic aldehyde oxidoreductase PaoABC from Escherichia coli: SAXS and preliminary X-ray crystallography analysis. Int J Mol Sci 15, 2223-2236 (2014).

19. Tullman-Ercek, D. et al. Export pathway selectivity of Escherichia coli twin arginine translocation signal peptides. J Biol Chem 282, 8309-8316 (2007).

20. Berks, B. C., Sargent, F. \& Palmer, T. The Tat protein export pathway. Mol Microbiol 35, 260-274 (2000).

21. Shanks, R. M., Caiazza, N. C., Hinsa, S. M., Toutain, C. M. \& O’Toole, G. A Saccharomyces cerevisiae-based molecular tool kit for manipulation of genes from gram-negative bacteria. Appl Environ Microbiol 72, 5027-5036 (2006).

22. Bogsch, E. G. et al. An essential component of a novel bacterial protein export system with homologues in plastids and mitochondria. J Biol Chem 273, 18003-18006 (1998).

23. Schagger, H., Cramer, W. A. \& von Jagow, G. Analysis of molecular masses and oligomeric states of protein complexes by blue native electrophoresis and isolation of membrane protein complexes by two-dimensional native electrophoresis. Anal Biochem 217, 220-230 (1994).

24. Waraho-Zhmayev, D., Gkogka, L., Yu, T. Y. \& DeLisa, M. P. A microbial sensor for discovering structural probes of protein misfolding and aggregation. Prion 7 , 151-156 (2013).

25. Wolf, E., Hofmeister, R., Kufer, P., Schlereth, B. \& Baeuerle, P. A. BiTEs: bispecific antibody constructs with unique anti-tumor activity. Drug Discov Today 10, 1237-1244 (2005)

26. der Maur, A. A. et al. Direct in vivo screening of intrabody libraries constructed on a highly stable single-chain framework. J Biol Chem 277, 45075-45085 (2002).

27. Koch, H., Grafe, N., Schiess, R. \& Pluckthun, A. Direct selection of antibodies from complex libraries with the protein fragment complementation assay. $J \mathrm{Mol} \mathrm{Biol}$ 357, 427-441 (2006).

28. Rachubinski, R. A. \& Subramani, S. How proteins penetrate peroxisomes. Cell 83, 525-528 (1995).

29. Jormakka, M., Tornroth, S., Byrne, B. \& Iwata, S. Molecular basis of proton motive force generation: structure of formate dehydrogenase-N. Science 295, 1863-1868 (2002).

30. Dubini, A. \& Sargent, F. Assembly of Tat-dependent [NiFe] hydrogenases: identification of precursor-binding accessory proteins. FEBS Lett 549, 141-146 (2003).

31. Leimkuhler, S. et al. Xanthine dehydrogenase from the phototrophic purple bacterium Rhodobacter capsulatus is more similar to its eukaryotic counterparts than to prokaryotic molybdenum enzymes. Mol Microbiol 27, 853-869 (1998).

32. Riethmuller, G. Symmetry breaking: bispecific antibodies, the beginnings, and 50 years on. Cancer Immun 12, 12 (2012).
33. May, C., Sapra, P. \& Gerber, H. P. Advances in bispecific biotherapeutics for the treatment of cancer. Biochem Pharmacol 84, 1105-1112 (2012).

34. Byrne, H., Conroy, P. J., Whisstock, J. C. \& O'Kennedy, R. J. A tale of two specificities: bispecific antibodies for therapeutic and diagnostic applications. Trends Biotechnol 31, 621-632 (2013).

35. Mack, M., Riethmuller, G. \& Kufer, P. A small bispecific antibody construct expressed as a functional single-chain molecule with high tumor cell cytotoxicity. Proc Natl Acad Sci U S A 92, 7021-7025 (1995).

36. Mallender, W. D. \& Voss, E. W., Jr. Construction, expression, and activity of a bivalent bispecific single-chain antibody. J Biol Chem 269, 199-206 (1994).

37. Kipriyanov, S. M. et al. Effect of domain order on the activity of bacterially produced bispecific single-chain Fv antibodies. J Mol Biol 330, 99-111 (2003).

38. Gruber, M., Schodin, B. A., Wilson, E. R. \& Kranz, D. M. Efficient tumor cell lysis mediated by a bispecific single chain antibody expressed in Escherichia coli. J Immunol 152, 5368-5374 (1994).

39. Zhang, Z. et al. Production of soluble and functional engineered antibodies in Escherichia coli improved by FkpA. Biotechniques 35, 1032-1038, 1041-1032 (2003).

40. Howard, P. L., Chia, M. C., Del Rizzo, S., Liu, F. F. \& Pawson, T. Redirecting tyrosine kinase signaling to an apoptotic caspase pathway through chimeric adaptor proteins. Proc Natl Acad Sci U S A 100, 11267-11272 (2003).

41. Whitaker, W. R., Davis, S. A., Arkin, A. P. \& Dueber, J. E. Engineering robust control of two-component system phosphotransfer using modular scaffolds. Proc Natl Acad Sci U S A 109, 18090-18095 (2012).

42. Alm, T., Yderland, L., Nilvebrant, J., Halldin, A. \& Hober, S. A small bispecific protein selected for orthogonal affinity purification. Biotechnol J 5, 605-617 (2010).

43. Tharad, M. et al. A three-hybrid system to probe in vivo protein-protein interactions: application to the essential proteins of the RD1 complex of $\mathrm{M}$ tuberculosis. PLoS One 6, e27503 (2011).

44. Vidal, M., Brachmann, R. K., Fattaey, A., Harlow, E. \& Boeke, J. D. Reverse twohybrid and one-hybrid systems to detect dissociation of protein-protein and DNA-protein interactions. Proc Natl Acad Sci U S A 93, 10315-10320 (1996).

45. Tirode, F. et al. A conditionally expressed third partner stabilizes or prevents the formation of a transcriptional activator in a three-hybrid system. J Biol Chem 272, 22995-22999 (1997).

46. Zhang, J. \& Lautar, S. A yeast three-hybrid method to clone ternary protein complex components. Anal Biochem 242, 68-72 (1996).

47. Karimova, G., Dautin, N. \& Ladant, D. Interaction network among Escherichia coli membrane proteins involved in cell division as revealed by bacterial twohybrid analysis. J Bacteriol 187, 2233-2243 (2005).

48. Fields, S. High-throughput two-hybrid analysis. The promise and the peril. FEBS J 272, 5391-5399 (2005)

49. von Mering, C. et al. Comparative assessment of large-scale data sets of proteinprotein interactions. Nature 417, 399-403 (2002).

50. Datsenko, K. A. \& Wanner, B. L. One-step inactivation of chromosomal genes in Escherichia coli K-12 using PCR products. Proc Natl Acad Sci U S A 97, 6640-6645 (2000).

51. Baba, T. et al. Construction of Escherichia coli K-12 in-frame, single-gene knockout mutants: the Keio collection. Mol Syst Biol 2, 20060008 (2006).

52. Santini, C. L. et al. Translocation of jellyfish green fluorescent protein via the Tat system of Escherichia coli and change of its periplasmic localization in response to osmotic up-shock. J Biol Chem 276, 8159-8164 (2001).

53. Oldenburg, K. R., Vo, K. T., Michaelis, S. \& Paddon, C. Recombination-mediated PCR-directed plasmid construction in vivo in yeast. Nucleic Acids Res 25, 451-452 (1997).

54. Karlsson, A. J. et al. Engineering antibody fitness and function using membrane-anchored display of correctly folded proteins. J Mol Biol 416, 94-107 (2012)

55. Tao, H. et al. Purifying natively folded proteins from inclusion bodies using sarkosyl, Triton X-100, and CHAPS. Biotechniques 48, 61-64 (2010).

56. Eubel, H., Braun, H. P. \& Millar, A. H. Blue-native PAGE in plants: a tool in analysis of protein-protein interactions. Plant Methods 1, 11 (2005).

\section{Acknowledgments}

We thank Andreas Plückthun for kindly providing plasmids encoding genes used in this study. This material is based upon work supported by the NSF Career Award CBET-0449080 (to M.P.D.), NIH Grant CA132223A (to M.P.D.) and 1R43GM093807-0 (to M.P.D.), New York State Office of Science, Technology and Academic Research Distinguished Faculty Award (to M.P.D.), NIH Chemical Biology Training Grant T32 GM008500 (fellowship to A.D.P.), and NSF GK-12 DGE-0841291 (fellowship to A.D.P.)

\section{Author contributions}

H.-C.L. and A.D.P. designed research, performed research, analyzed data, and wrote the paper. M.A.R. designed research, performed research, analyzed data. M.P.D. conceptualized project, designed research, analyzed data, and wrote the paper. All authors reviewed the manuscript. 


\section{Additional information}

Supplementary information accompanies this paper at http://www.nature.com/ scientificreports

Competing financial interests: The authors declare no competing financial interests.

How to cite this article: Lee, H.-C., Portnoff, A.D., Rocco, M.A. \& DeLisa, M.P. An engineered genetic selection for ternary protein complexes inspired by a natural

three-component hitchhiker mechanism. Sci. Rep. 4, 7570; DOI:10.1038/srep07570 (2014).

This work is licensed under a Creative Commons Attribution 4.0 International License. The images or other third party material in this article are included in the article's Creative Commons license, unless indicated otherwise in the credit line; if the material is not included under the Creative Commons license, users will need to obtain permission from the license holder in order to reproduce the material. To view a copy of this license, visit http://creativecommons.org/licenses/by/4.0/ 